\title{
Subclinical Needle Tract Seeding by Endoscopic Ultrasound-Guided Fine-Needle Aspiration for Pancreatic Cancer
}

\author{
Nozomi Uozumi Shoji Oura Shinichiro Makimoto \\ Department of Surgery, Kishiwada Tokushukai Hospital, Kishiwada, Japan
}

\section{Keywords}

Endoscopic ultrasonography-guided fine-needle aspiration - Endoscopic ultrasonography ·

Needle tract seeding · Pancreatic cancer

\begin{abstract}
A 77-year-old woman with epigastralgia was referred to our hospital. Abdominal computed tomography showed a hypointense mass in the pancreatic tail. Abdominal and endoscopic ultrasonography (EUS) showed a hypo-echoic mass, $25 \times 25 \mathrm{~mm}$ in size, with pancreatic duct dilatation. EUS-guided fine-needle aspiration (EUS-FNA) was performed to the mass through gastric posterior wall. Pathological examination showed atypical cells growing papillary or tubular fashion, leading to the diagnosis of adenocarcinoma. Under the preoperative diagnosis of T2NOMO pancreatic cancer, the patient underwent distal pancreatectomy and splenectomy. Macroscopic view of the resected specimen showed a presumed puncture-induced pancreatic pseudocyst adjacent to the pancreas. Pathological examination showed well-differentiated adenocarcinoma and a pseudocyst with presumed migrated atypical cells in the pseudocyst wall. The patient recovered uneventfully and has been on outpatient follow-up with adjuvant TS-1 therapy. Optimal treatment of pancreatic cancer naturally needs preoperative definitive diagnosis more strictly than other solid malignancies due to its much higher operative harm to the patients. EUS-FNA is a safe and effective diagnostic method but needs careful attention to the needle tract seeding.
\end{abstract}

\section{Karger $\stackrel{\text { ! }}{=}$}




\section{Introduction}

It is well known that early detection of malignant neoplasms can lead to higher probability of cure regardless of cancer types. Pancreatic cancer, however, rarely develops any symptoms until disease progression except for early pancreatic head cancer with jaundice [1]. Mass screening of the abdomen mainly consists of ultrasound evaluation and often fails to detect pancreatic disorder due to its deep location and the frequent presence of intestinal gas over the pancreas, easily leading to the late diagnosis of pancreatic cancer.

Endoscopic retrograde cholangiopancreatography (ERCP) [2] played important role in the diagnosis of pancreatic neoplasms for a long time. Magnetic resonance cholangiopancreatography [3], however, has almost completely taken place the position of ERCP. Percutaneous fine-needle aspiration (FNA) of the pancreatic lesions under ultrasound or computed tomography (CT) guidance has also been taken place by endoscopic ultrasound-guided FNA (EUS-FNA) [4, 5] since early 1990s. EUS-FNA is generally done through gastric posterior wall, causing perforation, infection, and bleeding at certain frequencies.

Needle tract seeding is sometimes observed in breast cancer after ultrasound-guided core needle biopsy and in lung cancer after CT-guided biopsy [6]. To prevent unfavorable outcomes due to these types of tumor seeding, surgeons have paid much attention to remove the puncture site of the breast skin in the operation and to minimize the interval between the biopsy day and the operation day. We here report a very rare case of pancreatic cancer showing pathologically proven needle tract tumor seeding just around the pancreas after EUS-FNA [7].

\section{Case Report}

A 77-year-old woman complaining of epigastralgia was referred to our hospital. Abdominal CT (Fig. 1a) incidentally showed a hypointense mass in the tail of the pancreas. Laboratory test showed no abnormal findings including carcinoembryonic antigen and CA19-9 levels. Abdominal and endoscopic ultrasound (EUS) showed a hypo-echoic mass, $32 \times 22 \mathrm{~mm}$ in size, accompanied by pancreatic duct dilatation (Fig. 2). EUS-FNA using a 22 gauge needle was performed to the mass twice through gastric posterior wall. Pathological examination of the biopsied specimen showed atypical cells growing papillary or tubular fashion, leading to the diagnosis of adenocarcinoma. Chest CT showed an oval mass, $24 \times 22 \mathrm{~mm}$ in size, in the left supraclavicular region. Positron emission tomography/CT showed significant (standard uptake value-max of 3.8) and faint fluorodeoxyglucose uptake in the pancreatic tail and left supraclavicular region, respectively. To exclude distant lymphatic metastasis, we initially tried to biopsy the left cervical mass and found the tumor to be a neurogenic tumor in the brachial plexus with no malignant findings on cytological study. Under the preoperative diagnosis of T2N0M0 pancreatic cancer, the patient underwent distal pancreatectomy and splenectomy. Macroscopic view of the resected specimen showed an old blood clot on the pancreatic anterior surface due to EUS-FNA (Fig. 1a) and a presumed puncture-induced pancreatic pseudocyst adjacent to the pancreas on cut surface. Pathological examination showed well-differentiated adenocarcinoma growing in papillary and tubular fashion, a pseudocyst without lining epithelial cells, and atypical cells with papillary or tubular growth pattern, i.e., similar to those of the main tumor, locating in the presumed iatrogenic pseudocyst wall (Fig. 3). The patient recovered uneventfully and was discharged on the 17th day after operation. Under the pathological stage\|A pancreatic cancer, the patient has been receiving postoperative adjuvant chemotherapy using TS-1 [8].

\section{Karger'}




\section{Case Reports in Oncology}

\begin{tabular}{l|l}
\hline Case Rep Oncol 2021;14:977-982 \\
\hline DOI: 10.1159/000516756 & $\begin{array}{l}\text { @ 2021 The Author(s). Published by S. Karger AG, Basel } \\
\text { www.karger.com/cro }\end{array}$ \\
\hline
\end{tabular}

Uozumi et al.: Subclinical Needle Tract Seeding by EUS-FNA for Pancreatic Cancer

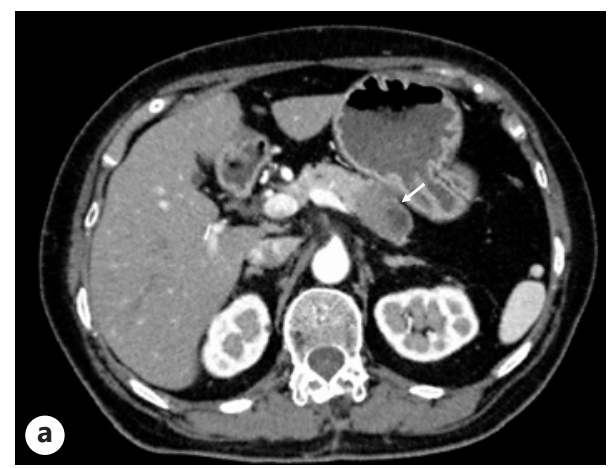

Fig. 1. CT findings. a CT showed a hypointense mass, 31 $\times 19 \mathrm{~mm}$ in size (arrow), in the pancreatic tail. Positron emission CT showed an uptake both in the pancreatic tail (b, arrow) and left supraclavicular region (c, arrow). CT, computed tomography.
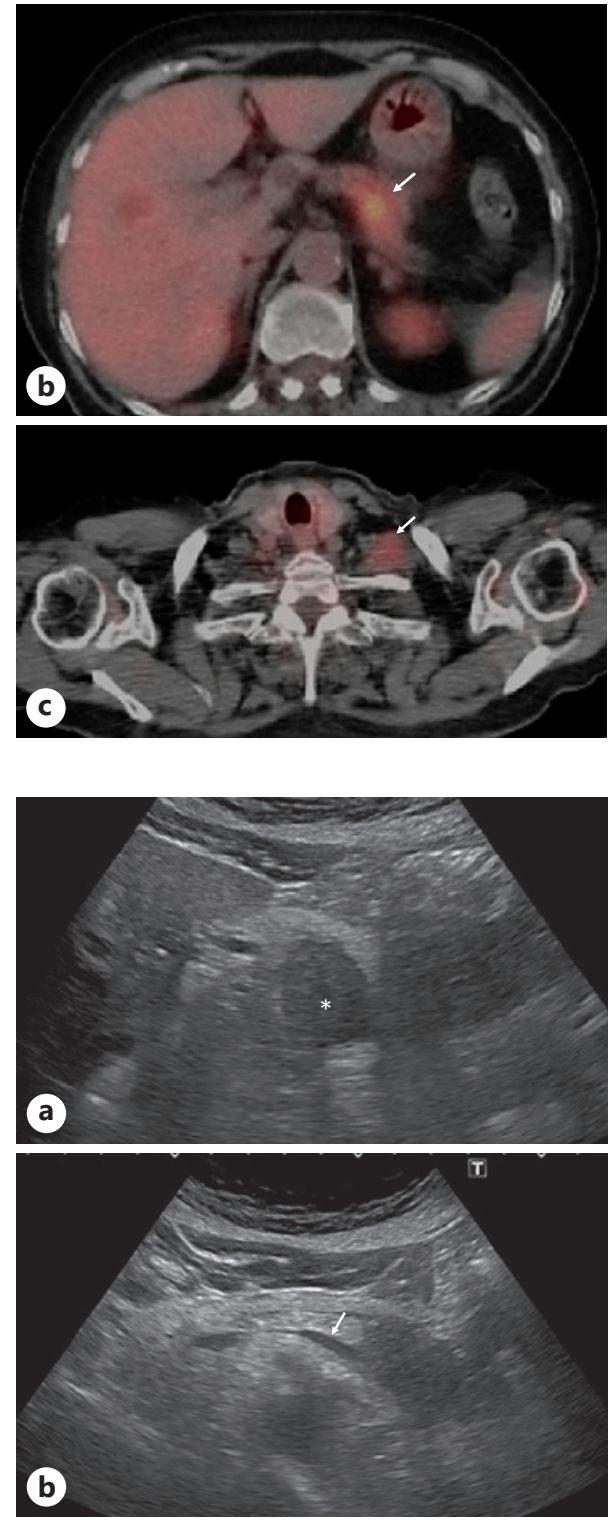

Fig. 2. Ultrasound findings. Ultrasound showed a hypoechoic mass, $32 \times 22 \mathrm{~mm}$ in size (a, asterisk), and pancreatic duct dilatation (b, arrow).

\section{Discussion}

Various solid malignancies can develop iatrogenic tumor cell migration, called needle tract seeding, through tumor cell harvesting such as core needle biopsy and FNA under ultrasound or CT guidance. However, local recurrence with needle tract seeding is extremely rare 


\section{Case Reports in Oncology}
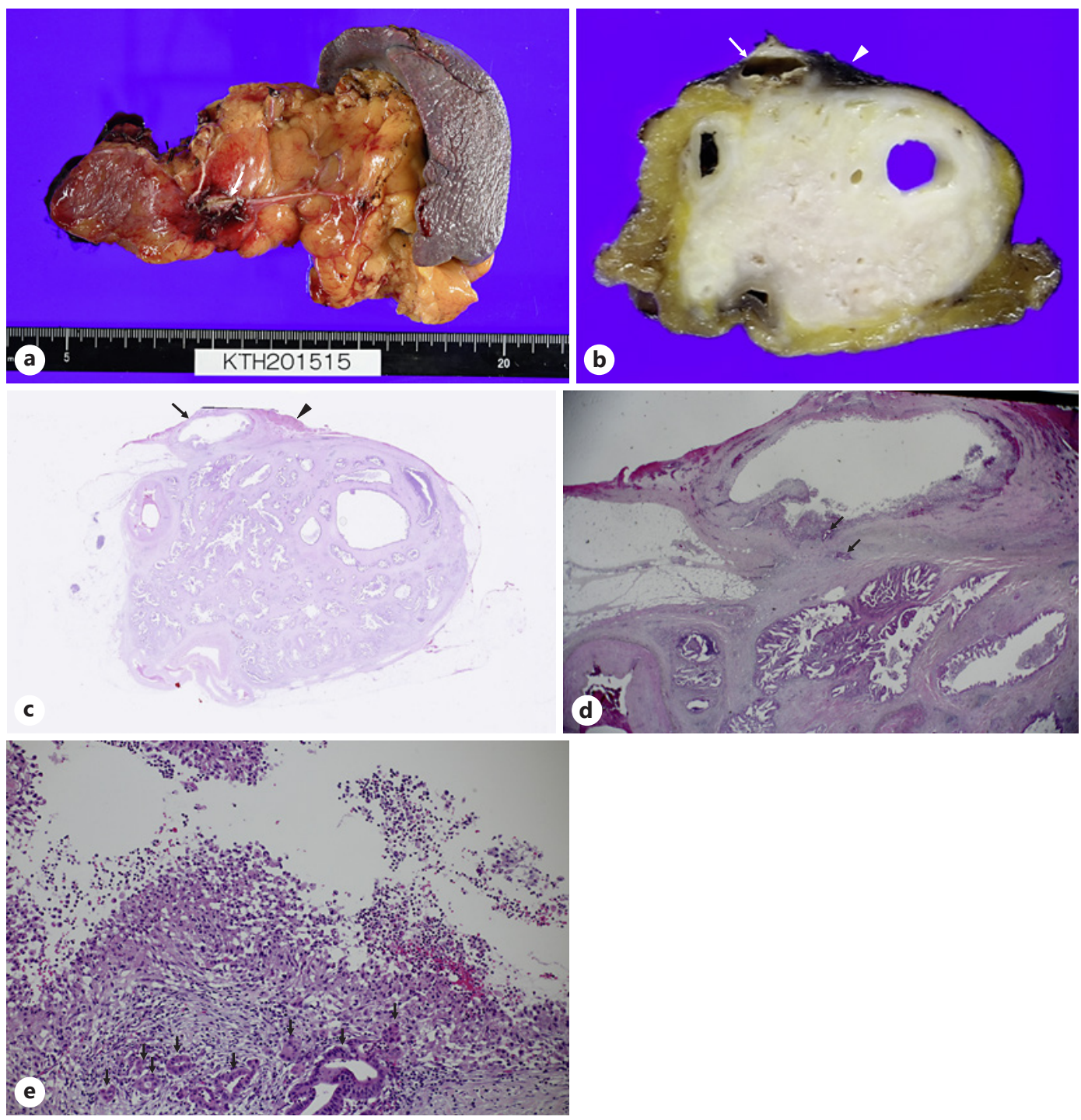

Fig. 3. Pathological findings. a Macroscopic view of the resected specimen showed an old blood clot (arrow) on the anterior surface of the pancreas. b Cut surface of the tumor showed a presumed pseudocyst (arrow) and old blood (arrowhead). c Low magnified view showed well-differentiated adenocarcinoma growing in papillary and tubular fashion and a pseudocyst (arrow) with adjacent erythrocytes (arrowhead). d High magnified view showed a pseudocyst without lining epithelial cells and atypical cell clusters (arrows) in the pseudocyst wall. e Higher magnified view showed atypical cell clusters (arrows) in the suppurative granuloma.

in breast cancer due mainly to antitumor effect of adjuvant radiotherapy to the conserved breast or chest wall. In addition, effective systemic therapies such as chemotherapy, endocrine therapy, and antihuman epidermal growth receptor type 2 therapy can further eradicate the mechanically migrated malignant cells. However, presumed pleural dissemination with needle tract seeding in lung cancer is often found after CT-guided biopsy of the malignant lung lesion.

Its diagnostic difficulties make the incidence of pancreatic cancer much higher in developed countries than in developing countries [9]. Image diagnosis of pancreatic cancer mainly consists of ultrasonography, computed tomography, magnetic resonance imaging, ERCP [2], and magnetic resonance cholangiopancreatography [3]. Optimal treatment of 
pancreatic cancer more strictly needs preoperative definitive diagnosis than other solid malignancies due to its much higher operative harm to the patients. Percutaneous needle punctures for pathological evaluation of the pancreatic lesion can damage many organs such as stomach, greater omentum, transverse colon, and small intestine, naturally leading to higher complication rates. EUS-FNA needs much shorter needle punctures than those of percutaneous techniques, suggesting safer pathological diagnosis.

Ikezawa et al. [10] reported the incidence of peritoneal carcinomatosis as 14.9 and $17.9 \%$ in patients examined with ERCP and EUS-FNA, respectively. Although some patients in EUS-FNA group might develop peritoneal carcinomatosis by needle tract seeding, recurrence of the pancreatic cancer by this mechanism thus far reported has been limited to single case reports with gastric wall recurrence, especially gastric posterior wall recurrence. This fact implies the difficulty of pathological verification of the correlation between the peritoneal carcinomatosis and needle tract seeding. However, its aggressive nature of pancreatic cancer should promote fluid-forming dissemination, that is, peritoneal carcinomatosis, of pancreatic cancer with needle tract seeding. In this case, presumed migrated malignant cells were observed in the wall of the pseudocyst formed by EUS-FNA procedure. To our knowledge, this is the 1st case of pathologically proven needle tract seeding with EUS-FNA just around the pancreas.

Minaga et al. [7] reported in their review article the characteristics of needle tract seeding from pancreatic cancer as follows: (1) a median interval from EUS-FNS to detection of needle tract seeding was 20 months, (2) most of the tumors was manifested as a submucosal tumor, (3) median tumor size was $30 \mathrm{~mm}$, and (4) pancreatic head cancer never developed needle tract seeding after EUS-FNA due to its operative method. Their report suggests that even in cases of protruding type mass formation more than half the cases of needle tract seeding recur within 2 years after EUS-FNA, and the needle tract resection is very important to prevent or minimize this type of recurrence. In this case, resection of the target lesion fortunately leads to the needle tract resection. It, therefore, is highly recommended that endoscopic sonographers perform EUS-FNA in a manner to include the needle tract in the expected resection field as much as possible and surgeons resect the needle tract.

Pancreatic cancer, once recurring, has an extremely poor prognosis. To maximally reduce the recurrence by the mechanism of needle tract seeding, physicians should take 2 possible measures: eradication of the migrated cancer cells and minimization of the effect of cancer cell seeding through needle puncture. In the lung cancer operation, thoracic surgeons often pour distilled water-bearing low osmotic pressure to the operative field to kill invisible disseminated cancer cells in the thorax after the completion of lung cancer operation. We can expect similar cell-killing effect with distilled water in the pancreatic cancer operation but must also take into account more serious adverse effects of distilled water on the gastrointestinal tract than those in the thoracic cavity. It, therefore, is feasible to minimize the time from EUS-FNA to the definitive operation and to resect the needle tract for the reduction of adverse effects of needle tract seeding. In conclusion, physicians should note the possibility of needle tract seeding in the histological diagnosis of pancreatic cancer.

\section{Statement of Ethics}

We have reported this case in compliance with the Declaration of Helsinki. Written informed consent was obtained from the patient for the publication of this case report and any accompanying images.

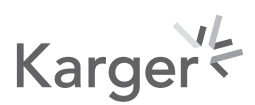




\section{Conflict of Interest Statement}

The authors have no conflicts of interest to declare.

\section{Funding Sources}

No funding was received for this research.

\section{Author Contributions}

N. Uozumi contributed to the design of the report. S. Oura drafted the manuscript. S. Makimoto revised the manuscript. All the authors have read and approved the final version of the manuscript.

\section{References}

1 Porta M, Fabregat X, Malats N, Guarner L, Carrato A, de Miguel A, et al. Exocrine pancreatic cancer: symptoms at presentation and their relation to tumour site and stage. Clin Transl Oncol. 2005;7:189-97.

2 Niederau C, Grendell JH. Diagnosis of pancreatic carcinoma. Imaging techniques and tumor markers. Pancreas. 1992; 7:66-86.

3 Adamek HE, Albert J, Breer H, Weitz M, Schilling D, Riemann JF. Pancreatic cancer detection with magnetic resonance cholangiopancreatography and endoscopic retrograde cholangiopancreatography: a prospective controlled study. Lancet. 2000;356:190-3.

4 Puli SR, Bechtold ML, Buxbaum JL, Eloubeidi MA. How good is endoscopic ultrasound-guided fine-needle aspiration in diagnosing the correct etiology for a solid pancreatic mass? A meta-analysis and systematic review. Pancreas. 2013;42:20-6.

5 Chen J, Yang R, Lu Y, Xia Y, Zhou H. Diagnostic accuracy of endoscopic ultrasound-guided fine-needle aspiration for solid pancreatic lesion: a systematic review. J Cancer Res Clin Oncol. 2012;138:1433-41.

6 DelMaschio A, Vanzulli A, Sironi S, Castrucci M, Mellone R, Staudacher C, et al. Pancreatic cancer versus chronic pancreatitis: diagnosis with CA 19-9 assessment, US, CT, and CT-guided fine-needle biopsy. Radiology. 1991; 178:95-9.

7 Minaga K, Takenaka M, Katanuma A, Kitano M, Yamashita Y, Kamata K, et al. Needle tract seeding: an overlooked rare complication of endoscopic ultrasound-guided fine-needle aspiration. Oncology. 2017;93(Suppl 1):107-12.

8 Ueno H, Ioka T, Ikeda M, Ohkawa S, Yanagimoto H, Boku N, et al. Randomized phase III study of gemcitabine plus S-1, S-1 alone, or gemcitabine alone in patients with locally advanced and metastatic pancreatic cancer in Japan and Taiwan: GEST study. J Clin Oncol. 2013;31:1640-8.

9 GBD 2017 Pancreatic Cancer Collaborators. The global, regional, and national burden of pancreatic cancer and its attributable risk factors in 195 countries and territories, 1990-2017: a systematic analysis for the Global Burden of Disease Study 2017. Lancet Gastroenterol Hepatol. 2019;4:934-47.

10 Ikezawa K, Uehara H, Sakai A, Fukutake N, Imanaka K, Ohkawa K, et al. Risk of peritoneal carcinomatosis by endoscopic ultrasound-guided fine needle aspiration for pancreatic cancer. J Gastroenterol. 2013;48:966-72.

\section{Karger'k}

